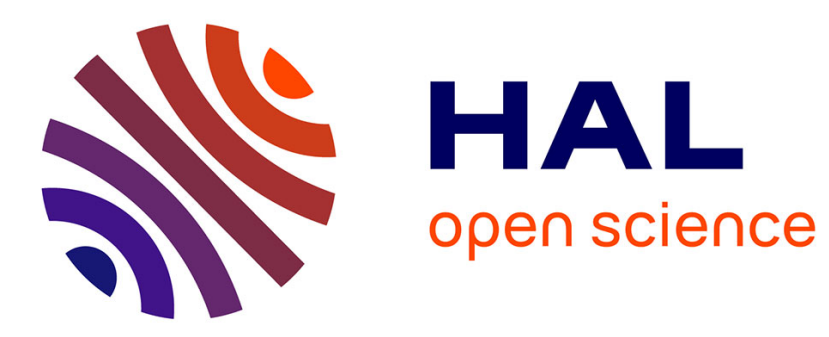

\title{
Optical Flow and Phase Portrait Methods for Environmental Satellite Image Sequences
}

\author{
Isaac Cohen, Isabelle Herlin
}

\section{To cite this version:}

Isaac Cohen, Isabelle Herlin. Optical Flow and Phase Portrait Methods for Environmental Satellite Image Sequences. Proceedings of European Conference on Computer Vision (ECCV), Apr 1996, Cambridge, United Kingdom. pp.141-150, 10.1007/3-540-61123-1_134 . inria-00626437

\section{HAL Id: inria-00626437 https://hal.inria.fr/inria-00626437}

Submitted on 20 Apr 2016

HAL is a multi-disciplinary open access archive for the deposit and dissemination of scientific research documents, whether they are published or not. The documents may come from teaching and research institutions in France or abroad, or from public or private research centers.
L'archive ouverte pluridisciplinaire HAL, est destinée au dépôt et à la diffusion de documents scientifiques de niveau recherche, publiés ou non, émanant des établissements d'enseignement et de recherche français ou étrangers, des laboratoires publics ou privés. 


\title{
Optical Flow and Phase Portrait Methods for Environmental Satellite Image Sequences
}

\author{
Isaac COHEN, Isabelle HERLIN \\ AIR Project, \\ INRIA, Rocquencourt \\ B.P. 105, 78153 Le Chesnay CEDEX, France. \\ Email Isaac.Cohen@inria.fr, Isabelle.Herlin@inria.fr
}

\begin{abstract}
We present in this paper a motion computation and interpretation framework for oceanographic satellite images. This framework is based on the use of a non quadratic regularization technique in optical flow computation that preserves flow discontinuities. We also show that using an appropriate tessellation of the image according to an estimate of the motion field can improve optical flow accuracy and yields more reliable flows. This method defines a non uniform multiresolution scheme that refines mesh resolution only in the neighborhood of moving structures. The second part of the paper deals with the interpretation of the obtained displacement field. We use a phase portrait model with a new formulation of the approximation of an oriented flow field. This allows us to consider arbitrary polynomial phase portrait models for characterizing salient flow features. This new framework is used for processing oceanographic and atmospheric image sequences and presents an alternative to the very complex physical modelling techniques.
\end{abstract}

Keywords: Optical flow, Non quadratic regularization, Finite element method, Adaptive mesh, Phase portrait, Flow pattern classification, Ocean circulation.

\section{Introduction}

Oceanographic and atmospheric images obtained from environmental satellite platforms present a new challenge for geosciences and computer vision. The wide ranges of remote sensors allow to characterize natural phenomena through different physical measurements. For example Sea Surface Temperature (SST), Altimetry and Ocean color can be used simultaneously for characterizing vortex structures in the ocean. A major advantage of environmental remote sensing is the regular sampling of the measurements and their availability. These regular temporal and spatial data samplings allow to characterize the short range evolution of atmospheric and oceanographic processes with image sequence processing.

In this paper we focus on dynamic satellite image analysis. The purpose of this paper is to derive a complete framework for processing large oceanographic and atmospheric image sequences in order to detect global displacements (oceanographic streams, clouds motion, ...), or to localize particular structures like vortices and fronts. These characterizations will help in initializing particular processes in a global monitoring system. 
Processing such an image sequence raise some specific problems. Indeed, computing an apparent motion field to characterize short range evolution must take into account discontinuities of the motion field that occur near SST temperature fronts and clouds' boundaries. For this purpose we make use of a new regularization method for solving the optical flow constraint equation which involves a non quadratic regularization allowing flow discontinuities. Furthermore, an important issue is the reduction of the numerical complexity due to the large size of the images (typically $2048 \times 1024$ ). The minimization model is handled through a finite element method allowing the use of a non uniform domain tessellation. This tessellation is obtained with a mesh subdivision model allowing to obtain, locally, a finer mesh resolution near moving structures. These structures are detected and localized by studying the norm of the estimated motion field along the direction of the image gradient. Using such a non uniform domain tessellation yields an accurate optical flow near moving structures and a lower numerical complexity.

The second part of the paper deals with the interpretation of the obtained displacement field. A velocity field can be studied from two different view points. First, it is a motion field and therefore it can be studied according to the analysis of fluid motion. On the other hand, it is a vector field whose topology is described by its critical points and its salient features. This approach is more suitable in our context since characterizing salient flow features will help us in locating interesting structures (like vortices and fronts) which represent physical phenomena appearing in SST and atmospheric image sequences. We present in this paper a new approach for approximating an orientation field and characterizing the stationary points of the trajectories obtained from an arbitrary polynomial phase portrait. Furthermore the model is always linear, independently of the polynomial representation.

\section{Non Quadratic Optical Flow Computation}

The differential techniques used for the computation of optical flow are based on the image flow constraint equation:

$$
\frac{d I(x, y, t)}{d t}=I_{x} u+I_{y} v+I_{t}=0,
$$

where the subscripts $x, y$ and $t$ represent the partial derivatives. This equation, based on the assumption that the image grey level remains constant, relates the temporal and spatial changes of the image grey level $I(x, y, t)$ at a point $(x, y)$ in the image to the velocity $(u, v)$ at that point [10]. Equation (1) is not suffcient for computing the image velocity $(u, v)$ at each point since the two velocity components are constrained by only one equation; this is the aperture problem. Therefore, most of the techniques use a regularity constraint that restrains the space of admissible solutions of equation (1) ([1] and references therein). This regularity constraint is generally quadratic and therefore enforces the optical flow field to be continuous and smooth. But, true discontinuities can occur in the optical flow and they are generally located on the boundary between two 
surfaces representing two objects with different movements. This type of discontinuity occurs for example on temperature front in SST images, and cloud boundary in atmospheric images. Recovering this discontinuity is necessary for further analysis of oceanographic and atmospheric images. Indeed, locating and tracking of temperature fronts in oceanographic images represent an accurate estimation of the oceanic surface circulation. These fronts are defined as regions where the temperature variation is high. An accurate computation of optical flow components near these regions must take into account the flow field discontinuity along the temperature front. For this purpose, we defined a non quadratic regularization scheme preserving flow discontinuities while insuring a unique solution of equation (1). Several authors [2, 3] proposed non-quadratic schemes for the motion field regularity constraint. These schemes are based on the Graduated Non Convexity method, and allow a computation of optical flow components that preserves discontinuity but can not handle non uniform tessellation of the image which is an important issue when dealing with very large image sequences. The proposed method makes use of the $L^{1}$ norm (defined by $|u|_{1}=\int|u|$ ) for the regularization constraint. The advantage of this norm is that the variation of expressions like $|u|_{1}$ produces singular distributions as coefficients (e.g. Dirac functions). This property allows to preserve sharp signals as well as discontinuities in the space of $L^{1}$ functions. Such a property can be used to constrain the set of admissible solutions of Eq. (1). Considering the space of functions with bounded variation, i.e.: $B V_{1}=\left\{f=\left(f_{1}, f_{2}\right)\right.$ such that $\left.\int_{\Omega}\left|\nabla f_{1}\right|+\left|\nabla f_{2}\right| d x d y<+\infty\right\}$, the optical flow problem can be stated as the minimization of the functional:

$$
\int_{\Omega} \sqrt{u_{x}^{2}+u_{y}^{2}}+\sqrt{v_{x}^{2}+v_{y}^{2}}+\left(I_{x} u+I_{y} v+I_{t}\right)^{2} d x d y
$$

where $u_{x}$ and $u_{y}$ (resp. $v_{x}$ and $v_{y}$ ) represent the partial derivatives of $u$ (resp. $v$ ) with respect to $x$ and $y$.

This minimization problem (2) can also be viewed as a constrained minimization problem where we search for a vector flow field in the space of functions with bounded variations $B V_{1}$, with the constraint of satisfying the optical flow stationarity equation (1).

The solution of the minimization problem (2) is obtained through the Euler - Lagrange equations:

$$
\left\{\begin{array}{l}
\mathcal{D} u+\left(u I_{x}^{2}+v I_{x} I_{y}+I_{x} I_{t}\right)=0 \\
\mathcal{D} v+\left(u I_{x} I_{y}+v I_{y}^{2}+I_{y} I_{t}\right)=0
\end{array}\right.
$$

where $\mathcal{D}$ is the nonlinear operator defined by:

$$
\mathcal{D} f=-\frac{\partial}{\partial x}\left(\frac{f_{x}}{\sqrt{f_{x}^{2}+f_{y}^{2}}}\right)-\frac{\partial}{\partial y}\left(\frac{f_{y}}{\sqrt{f_{x}^{2}+f_{y}^{2}}}\right) .
$$

Equation (3) is nonlinear and therefore must be processed in a particular way. An efficient method for solving this kind of nonlinear partial differential 
equations is to consider the associated evolution equations, or equivalently, the gradient descent method $[8,15]$. This time-dependent approach means that we solve the evolution equation:

$$
\left\{\begin{array}{l}
\frac{\partial u}{\partial t}+\mathcal{D} u+\left(u I_{x}^{2}+v I_{x} I_{y}+I_{x} I_{t}\right)=0 \\
\frac{\partial v}{\partial t}+\mathcal{D} v+\left(u I_{x} I_{y}+v I_{y}^{2}+I_{y} I_{t}\right)=0
\end{array}\right.
$$

and a stationary solution characterizes a solution of Eq. (3).

This evolution equation is solved through a finite element method allowing the use of arbitrary tessellations of the image domain by taking into account an index characterizing image motion in order to reduce the numerical complexity of the algorithm and increase its accuracy near moving structures (see Section 3).

\section{Mesh Subdivision}

Computing an optical flow field over an image sequence using a classical approach leads to the solution of a large set of linear equations in the case of the quadratic regularizer or to an iterative solution for the non quadratic case. In both approaches space discretization (i.e. image tessellation) is an important issue since it defines the accuracy of the solution and the numerical complexity of the algorithm. In this section we propose a selective multi-resolution approach. This method defines a new approach for coarse to fine grid generation. It allows to increment locally the resolution of the grid according to the studied problem. The advantage of such a method is its lowest numerical complexity and its higher accuracy. Each added node refines the grid in a region of interest and increases the numerical accuracy of the solved problem [4].

The method is based on a recursive subdivision of a given tessellation of the domain. This tessellation must fulfill the conform triangulation requirement of the FEM scheme [4], i.e.: any face of any $n$-simplex $\mathcal{T}_{1}$ in the triangulation is either a subset of the boundary of the domain, or a face of another n-simplex $\mathcal{T}_{2}$ in the triangulation. This requirement restrains the type of $\mathrm{n}$-simplex that can be used for an automatic non-uniform cell subdivision. We consider only triangular cells which are well adapted for domain triangulation and allow to derive a simple recursive subdivision scheme.

Given a triangulation $\mathcal{T}=\bigcup_{i} \mathcal{T}_{i}$ of the image domain, we have to refine it only near moving structures. These structures are characterized through $w_{\perp}$ representing the norm of the estimated motion field along the direction of the image gradient. Such a characterization is based on the image spatio-temporal gradients and does not require the knowledge of the optical flow field.

Let $I$ represents the image brightness and $\overrightarrow{\nabla I}$ its gradient vector field, then the optical flow equation (1) can be rewritten as [18]: $\frac{d I}{d t}=\frac{\partial I}{\partial t}+\|\nabla I\| w_{\perp}$ where $w_{\perp}$ is the norm of the component $\overrightarrow{w_{\perp}}$ of the motion field $\vec{w}=(u, v)$ along the direction of $\overrightarrow{\nabla I}$. If the flow constraint equation is satisfied (i.e. $\left.\frac{d I}{d t}=0\right)$ and $\|\nabla I\| \neq 0$, we obtain:

$$
\overrightarrow{w_{\perp}}=-\frac{\partial I / \partial t}{\|\nabla I\|} \cdot \frac{\nabla I}{\|\nabla I\|}
$$


representing the component of the motion field along the direction of the gradient in term of the partial derivatives of $I$. Although $w_{\perp}$ does not always characterize image motion due to the aperture problem, it allows to locate moving points. Indeed, $w_{\perp}$ is high near moving points and becomes null near stationary points.

The definition of $w_{\perp}$ gives the theoretical proof of the motion measure $D$ defined by Irani et al [11] and used by several authors [12, 17]:

$$
D(x, y, t)=\frac{\sum_{\left(x_{i}, y_{i}\right) \in W}\left|I\left(x_{i}, y_{i}, t+1\right)-I\left(x_{i}, y_{i}, t\right)\right|\left|\nabla I\left(x_{i}, y_{i}, t\right)\right|}{\sum_{\left(x_{i}, y_{i}\right) \in W}\left|\nabla I\left(x_{i}, y_{i}, t\right)\right|^{2}+C}
$$

where $W$ is a small neighborhood and $C$ a constant to avoid numerical instabilities. This motion measure, defined as residual motion, is a particular form of $w_{\perp}$ where the numerator and the denominator are summed over a small neighborhood.

The subdivision scheme is based on a splitting strategy. We start with a coarse tessellation of the image and split each cell $\mathcal{T}$ of the triangulation according to the norm $w_{\perp}$ summed all over the cell: $w_{\perp}^{\mathcal{T}}$. A cell is subdivided while $w_{\perp}^{\mathcal{T}}$ is greater than a given threshold and while its area is greater than another threshold. Furthermore, each subdivided cell must satisfy the conform triangulation requirement.

\section{Experimental Results}

We have experimented our method on the "Hamburg Taxi Sequence". In this street scene, there were four moving abjects at different speeds. We first construct an adaptive mesh from the spatio-temporal gradient according to the algorithm described in section 3. We set the motion threshold, i.e. $w_{\perp}^{\mathcal{T}}$, to one pixel/frame and the cell area threshold to 15 pixels. The obtained mesh and the optical flow field are displayed in figure 1 . We can observe that the mesh resolution is finer near moving objects allowing an important reduction of the algorithmic complexity, since we deal with 499 nodes and 956 triangles yielding an optical flow numerical accuracy lower than 0.5 pixel near moving structures. To achieve such an accuracy with a classical rectangular mesh, one has to consider 2560 nodes. The complete processing (i.e. the image gradients and the solution of Eq. (5)) takes 20 seconds on a Alpha 3000/500 workstation.

Our main objective is to derive the surface ocean circulation from a sequence of Sea Surface Temperature measurements. These infra-red measurements are the most reliable for surface motion estimation and are daily available through the NOAA satellites. These measurements are corrupted by the presence of clouds, consequently, the data usually considered are composite images obtained by considering at each image pixel the maximal value during a period of time. Figure 2 shows a SST image which concerns the confluence region near Argentina coasts where a combined stream is formed by the Falkland northward flowing current (cold water), and the southward flowing Brazilian current (hot water). The discontinuities are located in the regions where these two streams are combined (top of figure 2). The obtained displacement field and mesh used are also displayed. 

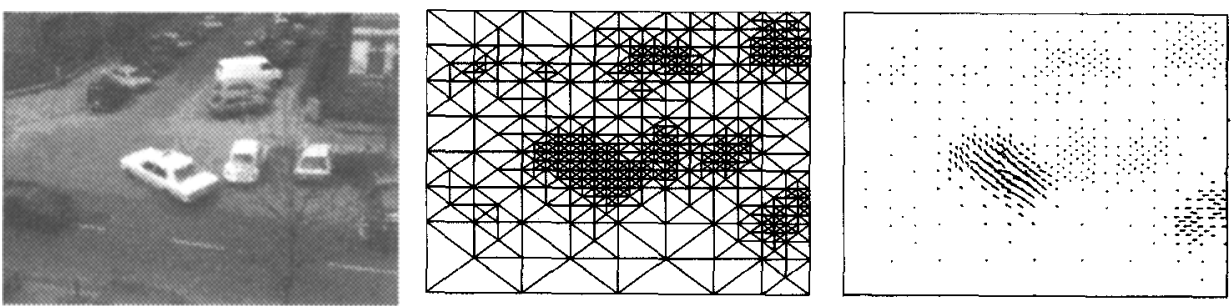

Fig. 1. Example of the use of an adaptive mesh to increase the numerical accuracy of the computed flow field while reducing the algorithmic complexity of the method. We display a frame of the Hamburg Taxi sequence, the associated mesh and the optical flow field.
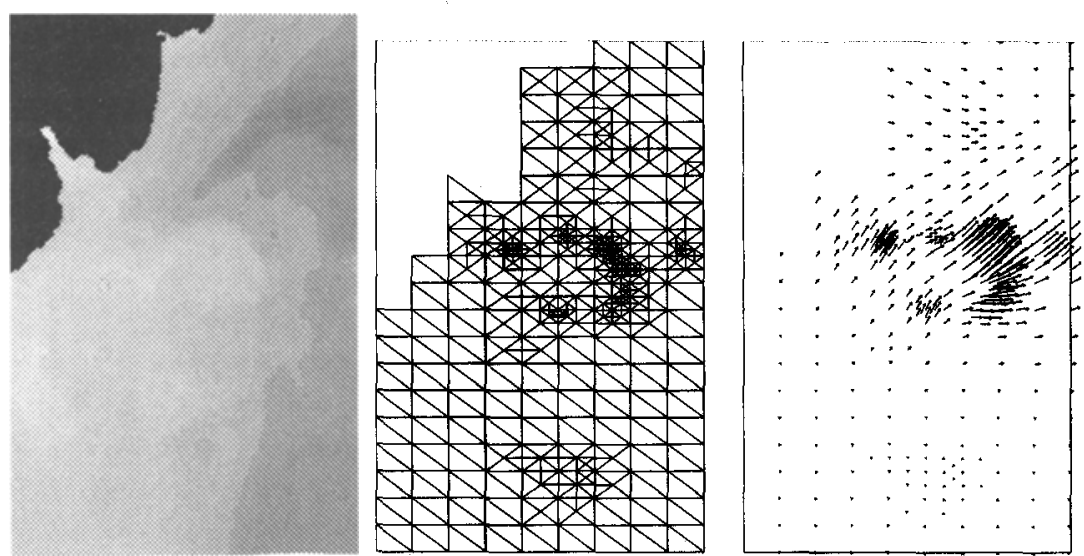

Fig. 2. An illustration of the adaptive mesh approach for computing optical flow components from an image sequence. This figure displays a frame of the SST image sequence of confluence region near Argentina coasts. In this region discontinuities are located in the regions where the streams are combined.

\section{Estimation of a Phase Portrait}

The computation of optical flow yields a quantitative measure of the flow field on each image point. By processing sea surface temperature and atmospheric image sequences we are also interested by the nature of motion since some phenomena like vortices are characterized through some specific patterns of the motion field. The extraction of higher level descriptors from a flow field is naturally crucial when studying fluid motion and vector analysis.

A classical approach is to use qualitative differential equations to characterize the orientation flow field by considering it as the velocity field of a particle in a dynamic system. Consider a particle governed by the model:

$$
g(x, y)=\left\{\begin{array}{l}
\frac{d X}{d t}=P(x, y) \\
\frac{d Y}{d t}=Q(x, y)
\end{array}\right.
$$


where $P(x, y)$ and $Q(x, y)$ are continuously differentiable functions. The particle trajectories are defined by the curves $(\varphi(t), \psi(t)), t>0$, satisfying: $(\varphi \prime(t), \psi \prime(t))=$ $(P(\varphi(t), \psi(t)), Q(\varphi(t), \psi(t)))$.

Modeling the orientation flow field by a dynamic system allows to characterize the flow field through the particles trajectories and their stationary points.

Different works were led on linear phase portrait models and their use for characterizing oriented texture fields $[14,16,19]$. The main drawback of a linear phase portrait is that it can handle only one critical point. Ford and Strickland [6] proposed a nonlinear phase portrait model allowing multiple critical point, but this model is computationally expensive and can not be generalized to arbitrary polynomials. This paper propose a new approach for approximating an orientation field and characterizing the stationary points of the trajectories obtained from an arbitrary polynomial phase portrait. Furthermore this model is always linear, independently of the polynomial representation.

Given two vectors $A$ and $B$, a distance between them may be given by [9]:

$$
\operatorname{dist}(A, B)=|A||B||\sin (\theta)|
$$

where $\theta$ is the angle subtended by the oriented segments. This distance represents also the area of the triangle formed by these oriented segments. Several authors $[7,14]$ used this measure to recover the six parameters of a linear phase model by minimizing locally the functional:

$$
S_{1}=\frac{1}{2} \sum_{i, j \in W}\left|A_{i j}\right|^{2}\left|B_{i j}\right|^{2}\left|\sin \left(\theta_{1 i j}-\theta_{2 i j}\right)\right|^{2},
$$

where $W$ is the local region over-which the linear approximation is searched, $\theta_{1}$ represents the linear model and $\theta_{2}$ the vector field to approximate.

The use the of Levenberg-Marquardt method for minimizing such a non quadratic functional, leads to a slow convergence rate [14]. In the following, we make use of another definition of the area subtended by two oriented segments that leads to the minimization of a quadratic functional. This other definition of the distance is obtained from the cross product of the vectors $A$ and $B$ :

$$
\operatorname{dist}(A, B)=\frac{1}{2}|A \times B| .
$$

Hence, considering a polynomial phase portrait model $g(x, y)$ defined by Eq. (8), where $P$ and $Q \in Q_{n}\left(\mathbb{R}^{2}\right)=\left\{p\right.$, st $\left.p(x, y)=\sum_{i, j \leq n} a_{i j} x^{i} y^{j}\right\}$, we fit the model to the given orientation field $f^{t}=\left(f_{1}, f_{2}\right)$ (obtained from the optical flow field framework) by minimizing locally:

$$
S_{2}(g)=\frac{1}{2} \sum_{i, j \in W}|f \times g|^{2},
$$

where $W$ is a neighborhood of the image point $(i, j)$. 
This criterion is easier to handle since it is quadratic. In [5] we have proved that recovering the coefficients of the two polynomials $P$ and $Q \in Q_{n}\left(\mathbb{R}^{2}\right)$ by minimizing $S_{2}$ amounts to an eigenvalue problem.

This new formulation of the phase portrait similarity measure allows to derive a linear algorithm characterizing arbitrary polynomial portraits.

\section{Flow Pattern Classification}

Analyzing a flow pattern consists in deriving a symbolic description from the model (8) fitted to the given local orientation field. In the case of optical flow, it would give a displacement information while in the case of fluid dynamic it will characterize the fluid velocity and the coherent structures of the flow. For example, the dynamic of the clouds can be tracked with infrared measurements, one can use such an approach in order to obtain a qualitative description of the atmospheric circulation without using a complex physical model of the underlying phenomena (see Figure 3).

The symbolic description of the trajectory of the phase portrait model was extensively used in the case of a linear model. In the general case (i.e. arbitrary polynomials) a classification of the stationary points can be obtained from the linearization of the polynomial model [13] but this assumes a location of critical points (i.e. to solve an arbitrary polynomial system). Instead we use the Index of a vector field to locate and characterize the stationary points. Let $g=(P, Q)$ be a vector field defined over a Jordan curve $J$ in the Euclidean plane, with no critical point on $J$. The index of $g$ over $J$ is proportional to the angular variation of the vector $g(M)$ (applied at $M \in J$ ) as $M$ describes $J$. For the system (8), the index over an oriented Jordan curve $J$ is given by:

$$
\operatorname{Index}(\mathrm{J})=\frac{1}{2 \pi} \oint_{J} d\left(\arctan \frac{Q}{P}\right)=\frac{1}{2 \pi} \oint_{J} \frac{P d Q-Q d P}{P^{2}+Q^{2}} .
$$

A classification of the flow field $g$ can be obtained by computing the index over a small circle surrounding an isolated critical point. Since the computation of the stationary points of system (8) leads to the problem of solving a system of polynomials with arbitrary degree, we choose to compute locally the index of $g=(P, Q)$ over the whole flow field: At each point we consider a circle contained in the centered window $W$ and we use the following classification:

- The index of a focus, a center or a node is equal to +1 ,

- The index of a saddle point is -1 .

Although this characterization is compendious, it characterizes the most important structures in a fluid flow field: the stationary points. The index measure, computed over all the flow, allows to obtain the critical points locations without computing the roots of the system (8). Once we locate these points we may use the linearization technique to obtain a complete description of the flow field in the neighborhood of its stationary points.

Figure 3 illustrates the complete framework for processing an infrared image sequence (Meteosat) of Europe (Courtesy of LMD). A frame of the sequence is 

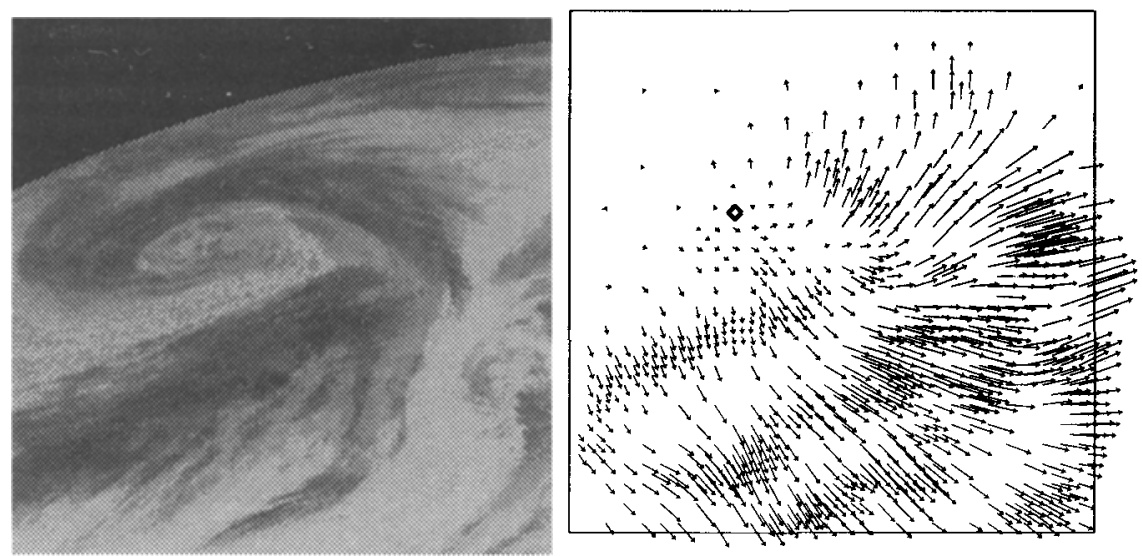

Fig. 3. A frame of the infrared image sequence, and the plot of the computed optical flow characterizing clouds motion. The black quadrangle represents the vortex detected with the index approach. In this case, the given flow field (i.e. the optical flow) was approximated with a $Q_{2}\left(\mathbb{R}^{2}\right)$ polynomial.

represented on the left part of the figure. The processing is done in two steps: A first step consists in computing the apparent motion field and a second one the characterizing the flow patterns. The right part of figure 3 , shows the optical flow obtained on a given frame. One can easily localize the vortex on the upper left corner of the figure from the flow structure. An approximation of this orientation field with a $Q_{2}\left(\mathbb{R}^{2}\right)$ polynomial phase portrait model and using the flow pattern classification described in section 6 gives an accurate localization of the vortex.

\section{Conclusion}

This research was done within the applicative context of environmental dynamic satellite images. We tried to solve some problem arising in processing a sequence of images representing the evolution of a physical phenomenon. This computer vision approach represents an alternative to the complex modeling of the underlying physical processes.

We proposed a two stages framework allowing the processing of environmental image sequences. The first stage concerns the efficient computation of an optical flow field that preserves flow discontinuities. The later one is concerned with the interpretation of the obtained displacement field.

We are currently studying the comparison between the computer vision approach and the classical method used by oceanographic and atmospheric researchers which deal with more elaborated models.

\section{References}

1. J.L. Barron, D.J. Fleet, and S.S. Beauchemin. Performance of optical flow techniques. International Journal of Computer Vision, 12(1):43-77, February 1994. 
2. M. J. Black. Recursive non-linear estimation of discontinuous flow fields. In Third European Conference on Computer Vision, pages 138-145, Sweden, May 1994. Springer-Verlag.

3. M.J. Black and P. Anandan. Robust dynamic motion estimation over time. In IEEE Proceedings of Computer Vision and Pattern Recognition, pages 296-302, June 1991.

4. P. G. Ciarlet. The finite element methods for elliptic problems. NORTHHOLLAND, Amsterdam, 1987.

5. I. Cohen and I. Herlin. A motion computation and interpretation framework for oceanographic satellite images. In IEEE, Computer Vision Symposium, pages 1318, Florida, November 1995.

6. R. M. Ford and R. N. Strickland. Nonlinear phase portrait models for oriented textures. In IEEE Proceedings of Computer Vision and Pattern Recognition, pages 644-645, New-York, June 1993.

7. R. M. Ford, R. N. Strickland, and B. A. Thomas. Image models for 2-D flow visualization and compression. CVGIP: Graphical models and Image Processing, 56(1):75-93, January 1994.

8. R. Glowinski. Numerical Methods for Nonlinear Variational Problems. SpringerVerlag, New-York, 1984. Springer Series in Computational Physics.

9. G. H. Golub and C. F. Van Loan. Matrix Computations. The Johns Hopkins University Press, London, second edition, 1989.

10. B.K.P. Horn and G. Schunck. Determining optical flow. Artificial Intelligence, 17:185-203, 1981.

11. M. Irani, B. Rousso, and S. Peleg. Detecting and tracking multiple moving objects using temporal integration. In Proceedings of the Second European Conference on Computer Vision 1992, pages 282-287, May 1992.

12. J.R. Muller, P. Anandan, and J.R. Bergen. Adaptive-complexity registration of images. In IEEE Proceedings of Computer Vision and Pattern Recognition, pages 953-957, 1994.

13. V. V. Nemytskii and V. V. Stepanov. Qualitative Theory of Differential Equations. Dover Publications, New York, 1989.

14. A. R. Rao and R. C. Jain. Computerized flow field analysis: Oriented textures fields. IEEE Transactions on Pattern Analysis and Machine Intelligence, 14(7):693-709, July 1992.

15. J.B. Rosen. The gradient projection method for nonlinear programming. Part I: Linear constraints. J. Soc. Indust. Appl. Math., 8(1):181-217, March 1960.

16. C.-F. Shu and R.C. Jain. Vector field analysis for oriented patterns. In IEEE Proceedings of Computer Vision and Pattern Recognition, pages 673-676, Urbana Champaign, Illinois, June 1992.

17. R. Szeliski and H.Y. Shum. Motion estimation with quadtree splines. Technical report, DEC Cambridge Research Lab, March 1995.

18. A. Verri and T. Poggio. Motion field and optical flow: Qualitative properties. IEEE Transactions on Pattern Analysis and Machine Intelligence, 11(5):490-498, May 1989.

19. J. Zhong, T.S. Huang, and R.J. Adrian. Salient structure analysis of fluid flow. In IEEE Proceedings of Computer Vision and Pattern Recognition, pages 310-315, Seattle, Washington, June 1994. 